\title{
Regularity at the Boundary for Solutions of Nonlinear Subelliptic Equations
}

\author{
Donatella DANielli
}

\begin{abstract}
In this article we establish an estimate, in terms of subelliptic $p$-capacity, for the modulus of continuity at the boundary of the solution to the Dirichlet problem associated to a class of subelliptic quasilinear equations. We infer from it the sufficiency part of a Wiener type criterion for the regularity of boundary points.
\end{abstract}

1. Introduction. Over the last decade, the study of nonelliptic equations arising from general families of non-commuting vector fields has undergone a great development. In spite of the formidable progress, there is still much to discover concerning the basic properties of solutions to these classes of equations. Consider a family of $C^{\infty}$ vector fields $X_{1}, \ldots, X_{m}$ in $\mathbf{R}^{n}, n \geq 3$, and assume that Hörmander's finite rank condition $[\mathrm{H}]$ is satisfied

$$
\operatorname{rank} \operatorname{Lie}\left[X_{1}, \ldots, X_{m}\right]=n
$$

at each $x \in \mathbf{R}^{n}$. In this note we are concerned with a class of nonlinear equations that naturally arise in the study of quasiregular mappings on stratified Lie groups, also known as Carnot groups [P]. Our purpose is to establish a quantitative estimate at the boundary for the solution to the Dirichlet problem. The model equation we have in mind is the so-called subelliptic $p$-Laplacian

$$
\sum_{j=1}^{m} X_{j}^{*}\left(|X u|^{p-2} X u\right)=0,
$$

where $X_{j}^{*}$ denotes the formal adjoint of $X_{j}, X u=\left(X_{1} u, \ldots, X_{m} u\right)$ is the subelliptic gradient of $u$ and $1<p<\infty$ is fixed. We stress that (1.1) is the Euler- 
Lagrange equation of the Sobolev functional

$$
J_{p}(u)=\int|X u|^{p} d x .
$$

When $p=2,(1.1)$ reduces to the Hörmander type equation

$$
\sum_{j=1}^{m} X_{j}^{*} X_{j} u=0 .
$$

A major achievement in the study of (1.2) came in 1985 with Nagel, Stein and Wainger's famous paper [NSW], in which the following estimate for the CarnotCaratheodory metric balls was proved: For every $K \Subset \mathbf{R}^{n}$ there exist positive constants $C, R_{0}$ and $Q$ such that, for any $x \in K, 0<r \leq R_{0}$, and $0<t<1$,

$$
\left|B_{d}(x, t r)\right| \geq C t^{Q}\left|B_{d}(x, r)\right| .
$$

Here and in the sequel, $B_{d}(x, r)=\left\{y \in \mathbf{R}^{n} \mid d(x, y)<r\right\}$ is the ball relative to the control distance $d$ associated to the vector fields $X_{1}, \ldots, X_{m}$. We note that (1.3) constitutes an ad hoc substitute for rescaling. The number $Q$ plays the role of a dimension in the local analysis of (1.2). It will be called the homogeneous dimension of $K$ with respect to the family $X_{1}, \ldots, X_{m}$. It is (1.3), together with the pointwise behaviour of the fundamental solutions of (1.2), found independently in $[\mathrm{SC}]$ and $[\mathrm{NSW}]$, that has made possible the development mentioned above.

Throughout the paper we will work with a different family of "balls" $B(x, r)$, which are better fitted to the subelliptic geometry. The latter are defined as properly rescaled level sets of the fundamental solution $\Gamma(x, y)$ of $(1.2)$; see (2.3) below. Among their main features we note that they are equivalent to the metric balls $B_{d}(x, r)$, and, furthermore, they support appropriate cut-off functions.

One important motivation for the study of equation (1.1) comes from the theory of quasiregular and quasiconformal mappings on stratified, nilpotent Lie groups; see [KR1], [KR2], [P], [HH]. In this connection, a relevant question is that of the boundary behaviour of solutions to the Dirichlet problem. In this paper we give a sufficient condition, in terms of subelliptic $p$-capacity, for the regularity of a boundary point, see Theorem 3.8 below. The latter is, in fact, a corollary of Theorem 3.1 which also implies the Hölder continuity at the boundary for solutions to the Dirichlet problem.

When $m=n$ and $X_{j}=\partial / \partial x_{j}$, our result recovers the sufficient part of Wiener's criterion for the standard $p$-Laplacian, proved by Maz'ya in [M]. Our approach is based on the interesting adaptation of $[\mathrm{M}]$ given in $[\mathrm{HKM}]$. Yet, despite the similarities, several basic questions have only recently been settled and many are still left open. We mention, for instance, the subelliptic Sobolev embedding with sharp exponents and the Harnack inequality; see [CDG1], [CDG2]. In this respect we point out that, working considerably harder, we could have 
treated a wider class of equations in the spirit of the results in [GZ]. However, in order to keep the presentation as simple as possible, we have chosen to confine the discussion to the significant class (2.1).

It is worth noting that our result finds an interesting application to the class of nontangentially accessible domains with respect to the Carnot-Carathéodory metric.

The plan of the paper is as follows. In Section 2, we collect most of the results that constitute the building blocks of the paper. Section 3 contains the proof of the main theorem.

Finally, we would like to thank the referee for his/her careful reading of the paper and for several valuable comments.

2. Some preliminary results. In this section, we introduce some definitions and results that will be needed in the sequel. We consider the equation

$$
\sum_{j=1}^{m} X_{j}^{*} A_{j}(x, X u)=0,
$$

where $A=\left(A_{1}, \ldots, A_{m}\right): \mathbf{R}^{n} \times \mathbf{R}^{m} \rightarrow \mathbf{R}^{m}$ is a measurable function satisfying the following assumptions: The mapping $x \rightarrow A(x, \xi)$ is measurable for all $\xi \in$ $\mathbf{R}^{m}$, the mapping $\xi \rightarrow A(x, \xi)$ is continuous for a.e. $x \in \mathbf{R}^{n}$ and, moreover, there exist $p \in(1, \infty)$ and $C_{0}>0$ such that

$$
\begin{aligned}
& |A(x, \xi)| \leq C_{0}|\xi|^{p-1}, \\
& A(x, \xi) \cdot \xi \geq|\xi|^{p}, \\
& A(x, t \xi)=t|t|^{p-2} A(x, \xi), \\
& (A(x, \xi)-A(x, \zeta)) \cdot(\xi-\zeta)>0,
\end{aligned}
$$

for every $x \in \mathbf{R}^{n}, \xi, \zeta \in \mathbf{R}^{m}, \xi \neq \zeta$ and $t \in \mathbf{R}-\{0\}$. Clearly, when

$$
A_{j}(x, \xi)=A_{j}(\xi)=|\xi|^{p-2} \xi_{j}, \quad j=1, \ldots, m,
$$

one recovers Equation (1.1).

Solutions to (2.1) shall be interpreted in a suitable weak sense. Throughout the paper $\Omega$ will denote a bounded open set in $\mathbf{R}^{n}$ and $Q$ will be the homogeneous dimension of $\Omega$ relative to $X_{1}, \ldots, X_{m}$.

Let $S^{1, p}(\Omega)$ (respectively $\stackrel{\circ}{S}^{1, p}(\Omega)$ ) denote the completion of the set

$$
\left\{u \in \operatorname{Lip}(\Omega)\left(\operatorname{resp} \operatorname{Lip}_{0}(\Omega)\right) \mid\|u\|_{S^{1, p}(\Omega)}<\infty\right\}
$$

in the norm

$$
\|u\|_{S^{1, p}(\Omega)}=\left[\int_{\Omega}\left(|u|^{p}+|X u|^{p}\right) d x\right]^{1 / p} .
$$


We say that $u \in S^{1, p}(\Omega)$ is a (weak) solution of (2.1) if

$$
\sum_{j=1}^{m} \int_{\Omega} A_{j}(x, X u) X_{j} \varphi d x=0
$$

for each $\varphi \in \stackrel{\circ}{S}^{1, p}(\Omega)$. At this point, it is worth emphasizing the link between the metric balls $B_{d}(x, r)$ and appropriate level sets of the fundamental solution of (1.2). One of the main results in [NSW] is the following: For every bounded set $U \subset \mathbf{R}^{n}$ there exist $C>0$ and $R_{0}>0$, such that for any $x \in U$ and $0<r \leq R_{0}$,

$$
C \Lambda(x, r) \leq\left|B_{d}(x, r)\right| \leq C^{-1} \Lambda(x, r),
$$

where $\Lambda(x, r)$ is a polynomial function in $r$ with nonnegative coefficients. In particular, the degree of $\Lambda(x, r)$ is bigger than or equal to $n$ and less than or equal to $Q$. Thereby, the function $r \mapsto \Lambda(x, r) / r^{2}$ is increasing. We let

$$
E(x, r)=\frac{\Lambda(x, r)}{r^{2}}
$$

and denote by $F(x, r)$ its inverse. We now introduce the sets

$$
B(x, r)=\left\{y \in \mathbf{R}^{n} \mid \Gamma(x, y)>\frac{1}{E(x, r)}\right\},
$$

where $\Gamma(x, y)$ is the fundamental solution of (1.2). The following facts were proved in [CGL]:

$$
\begin{aligned}
& B_{d}\left(x, \frac{r}{a}\right) \subseteq B(x, r) \subseteq B_{d}(x, a r), \\
& C d(x, y) \leq F\left(x, \Gamma(x, y)^{-1}\right) \leq C^{-1} d(x, y),
\end{aligned}
$$

for every $x \in K \Subset \Omega, y$ such that $d(x, y) \leq R_{0}$, with $C, R_{0}$, and $a$ depending on $K$. We stress that despite the notation, the sets $B(x, r)$ are not balls in a metric. Nonetheless, thanks to (2.4), (2.5), they can be effectively used as such. For our purposes it is important to observe that (1.3) continues to hold with the metric balls $B_{d}(x, r)$ replaced by the sets $B(x, r)$, namely,

$$
|B(x, t r)| \geq C t^{Q}|B(x, r)|,
$$

whenever $x \in K \subset \Omega, 0<r \leq R_{0}, 0<t<1$.

The following important result, proved in [CGL], provides the strongest motivation for the introduction of the sets $B(x, r)$.

Lemma 2.1. Let $K \Subset \Omega$. There exists $R_{0}>0$ such that given any $B(x, s) \subset B(x, t) \subset K$, with $t \leq R_{0}$, one can find $\varphi \in C_{0}^{\infty}(B(x, t))$ with $\varphi \equiv 1$ in $B(x, s)$ and

$$
|X \varphi| \leq \frac{C}{t-s} .
$$

Here, $C>0$ is independent of $x, s$, and $t$. 
In the sequel we shall need some results from [D], [CDG1], [CDG2].

We begin with recalling the notion of metric fractional integration operator of order $\alpha \in(0, Q]$ :

$$
I_{\alpha}(f)(y)=\int_{B_{R}}|f(\xi)| \frac{d(y, \xi)^{\alpha}}{|B(y, d(y, \xi))|} d \xi,
$$

where $B_{R}=B(x, R) \subset \Omega$ and $y \in B_{R}$. The following $L^{p}-$ continuity result holds.

Theorem 2.1. Let $0<\alpha<Q$ and $1 \leq p \leq \infty$.

(i) If $p>1$, then $I_{\alpha}$ maps $L^{p}\left(B_{R}\right)$ continuously into $L^{q}\left(B_{R}\right)$, with $0 \leq 1 / p-$ $1 / q \leq \alpha / Q$. Moreover, there exist $C>0$ and $R_{0}>0$ such that for any $R \leq R_{0}$, we have

$$
\left(\frac{1}{\left|B_{R}\right|} \int_{B_{R}}\left|I_{\alpha}(f)(x)\right|^{q} d x\right)^{1 / q} \leq C R^{\alpha}\left(\frac{1}{\left|B_{R}\right|} \int_{B_{R}}|f(x)|^{p} d x\right)^{1 / p}
$$

for every $f \in L^{p}\left(B_{R}\right)$.

(ii) $I_{\alpha}$ maps continuously $L^{1}\left(B_{R}\right)$ into $L^{Q /(Q-\alpha), \infty}\left(B_{R}\right)$. Moreover, there exist $C>0$ and $R_{0}>0$ such that for any $R \leq R_{0}, f \in L^{1}\left(B_{R}\right)$ and $\lambda>0$,

$$
\left|\left\{x \in B_{R} \mid I_{\alpha}(f)(x)>\lambda\right\}\right| \leq C \lambda^{-q} R^{\alpha Q}\left|B_{R}\right|^{1-q}\|f\|_{L^{1}\left(B_{R}\right)}^{q},
$$

where $q=Q /(Q-\alpha)$.

Theorem 2.1 is the first crucial step in proving the geometric Sobolev embedding.

Theorem 2.2. Let $U \subset \mathbf{R}^{n}$ be a bounded set. There exist two positive constants $C$ and $R_{0}$ such that for any $x_{0} \in U, B_{R}=B\left(x_{0}, R\right)$, with $0<R \leq R_{0}$, and any $u \in \stackrel{\circ}{S}^{1,1}\left(B_{R}\right)$ one has

$$
\left(\frac{1}{\left|B_{R}\right|} \int_{B_{R}}|u|^{k} d x\right)^{1 / k} \leq C R\left(\frac{1}{\left|B_{R}\right|} \int_{B_{R}}|X u| d x\right),
$$

where $1 \leq k \leq Q /(Q-1)$.

It is worth noting that from Theorem 2.2 one can easily infer the embedding

$$
\stackrel{\circ}{S}^{1, p}(\Omega) \hookrightarrow L^{p Q /(Q-p)}(\Omega)
$$

for all $p \in[1, Q)$. An important consequence of $(2.8)$ is the fact that a subset of $\mathbf{R}^{n}$ of vanishing $(X, p)$-capacity (see Definition 2.1 . below) has also zero Lebesgue measure.

Finally, we have the following result: 
Theorem 2.3. (Harnack Inequality) Let $1<p \leq Q$ and assume that $U \subset$ $\mathbf{R}^{n}$ is a bounded open set. Let $u \in S^{1, p}(U)$ be a nonnegative solution to (2.1). Then there exist $C>0$ and $R_{0}>0$ such that for each $B_{R}=B(x, R), B_{4 R} \subseteq U$ and $R \leq R_{0}$,

$$
\underset{B_{R}}{\operatorname{esssup}} u \leq \underset{B_{R}}{\operatorname{essinf}} u \text {. }
$$

From Theorem 2.3 one can infer, in a classical fashion, the Hölder continuity of solutions with respect to the $\left(X_{1}, \ldots, X_{m}\right)$-control distance:

Theorem 2.4. Let $u \in S^{1, p}(U)$ be a weak solution to (2.1), and suppose $\operatorname{esssup}_{U}|u|=M<\infty$. Then, there exist $C>0$ and $0<\alpha<1$, depending on $U$ and $M$, such that

$$
\operatorname{esssup}_{x, y \in U} \frac{|u(x)-u(y)|}{d(x, y)^{\alpha}} \leq C .
$$

Let us draw some consequences of these theorems. We omit their proofs, since they are essentially a step by step imitation of classical arguments. The first one is a maximum principle for solutions of (2.1).

Theorem 2.5. Let $u \in S^{1, p}(\Omega)$ be a solution of (2.1). Then either $u$ is constant in $\Omega$ or it cannot attain its maximum or minimum in $\Omega$.

The next result is a comparison lemma for solutions of (2.1).

Lemma 2.6. Let $u, v \in S^{1, p}(\Omega)$ be solutions of the equation (2.1). Assume $\min (u-v, 0) \in \stackrel{\circ}{S}^{1, p}(\Omega)$. Then $u \geq v$ a.e. in $\Omega$.

Finally, the structural conditions (2.2) imply that solutions of (2.1) enjoy the quasiminimizing property, as stated in the following lemma.

Lemma 2.7. Let $u \in S^{1, p}(\Omega)$ be a solution to (2.1) and assume $u-\varphi \in$ $\stackrel{\circ}{S}^{1, p}(\Omega)$. Then

$$
\int_{\Omega}|X u|^{p} d x \leq C_{0}^{p} \int_{\Omega}|X \varphi|^{p} d x
$$

where $C_{0}$ is as in $(2.2)$.

We now turn our attention to some fine properties of the subelliptic Sobolev spaces $S^{1, p}(\Omega)$. At this point, we introduce the notion of subelliptic $p$-capacity.

Definition 2.1. Let $K \subset \Omega$ be a compact set. We let

$$
\operatorname{cap}_{p}(K, \Omega)=\inf \left\{\int_{\Omega}|X u|^{p} d x \mid u \in C_{0}^{\infty}(\Omega), u \geq 1 \text { in } K\right\} \text {. }
$$

The $(X, p)$-capacity of an arbitrary set $E \subseteq \Omega$ in $\Omega$ is

$$
\operatorname{cap}_{p}(E, \Omega)=\inf _{\substack{G \subseteq \Omega \text { open } \\ E \subseteq G}} \sup _{K \text { compact }} \operatorname{cap}_{p}(K, \Omega) .
$$


The following definition will also be needed.

Definition 2.2. Let $B$ be any ball containing $\bar{\Omega}$.

(i) We say that $u: \Omega \rightarrow \mathbf{R} \cup\{\infty\}$ is $(X, p)$-quasicontinuous in $\Omega$ if for every $\varepsilon>0$ there exists an open set $G \subseteq \Omega$ such that $\operatorname{cap}_{p}(G, B)<\varepsilon$ and $\left.u\right|_{\Omega \backslash G}$ is real-valued and continuous;

(ii) A sequence of functions $\psi_{j}: \Omega \rightarrow \mathbf{R}$ is said to converge $(X, p)$-quasiuniformly in $\Omega$ to a function $\psi$ if for every $\varepsilon>0$ there exists an open set $G \subseteq \Omega$ such that $\operatorname{cap}_{p}(G, B)<\varepsilon$ and $\psi_{j} \rightarrow \psi$ uniformly in $\Omega \backslash G$.

The first result is a characterization of elements of $S^{1, p}(\Omega)$ in terms of $(X, p)$ quasicontinuous functions. Its proof is a generalization to the subelliptic context of the one in $[\mathrm{F}]$.

Theorem 2.8. Let $\left\{\varphi_{j}\right\}$ be a sequence of functions in $C(\Omega) \cap S^{1, p}(\Omega)$ and assume it is Cauchy in $S^{1, p}(\Omega)$. Then there exists a subsequence $\left\{\varphi_{k_{j}}\right\}$ converging $(X, p)$-quasiuniformly to a function $u \in S^{1, p}(\Omega)$.

An immediate consequence of Theorem 2.8 is the following.

Corollary 2.9 Let $u \in S^{1, p}(\Omega)$. Then there exists a $(X, p)$-quasicontinuous function $v \in S^{1, p}(\Omega)$ such that $u=v$ a.e.

The last result in this section is a characterization of functions in $\stackrel{\circ}{S}^{1, p}(\Omega)$ in terms of $(X, p)$-quasicontinuity. In the Euclidean context it is due to Bagby $[\mathrm{B}]$. We recall that a property is said to hold $(X, p)$-quasieverywhere if it holds except on a set of $(X, p)$-capacity zero and that $\Omega^{c}$ denotes the complement of $\Omega$ in $\mathbf{R}^{n}$.

Theorem 2.10. Let $u \in S^{1, p}(\Omega)$. Then $u \in \stackrel{\circ}{S}^{1, p}(\Omega)$ if, and only if, there exists a $(X, p)$-quasicontinuous function $v$ defined on all of $\mathbf{R}^{n}$ such that $v=u$ a.e. in $\Omega$ and $v=0(X, p)$-quasieverywhere in $\Omega^{c}$.

3. Boundary behaviour of Dirichlet functions. The main result in this section is an estimate, in terms of $(X, p)$-capacity, of the modulus of continuity at boundary points for solutions of the Dirichlet problem relative to the equation (2.1). Namely, we have the following.

Theorem 3.1. Let $1<p \leq Q$ and $f \in S^{1, p}(\Omega) \cap C(\bar{\Omega})$. Consider the solution $u$ of the Dirichlet problem

$$
\left\{\begin{array}{l}
\sum_{j=1}^{m} X_{j}^{*} A_{j}(x, X u)=0, \\
u-f \in \stackrel{\circ}{S^{1, p}}(\Omega),
\end{array}\right.
$$


and let $x_{0} \in \partial \Omega, R_{0} \geq 0,0<r \leq \rho \leq R_{0} / 2$. Then there exists a constant $C>0$, depending only on the vector fields $X_{1}, \ldots, X_{m}$, on $\Omega, p$ and $C_{0}$ (here $p$ and $C_{0}$ are as in (2.2)), such that

$$
\begin{aligned}
\operatorname{osc}\left(u, \Omega \cap B\left(x_{0}, r\right)\right) \leq \operatorname{osc}\left(f, \partial \Omega \cap \overline{B\left(x_{0}, 2 \rho\right)}\right) \\
\quad+\operatorname{osc}(f, \partial \Omega) \exp \left(-C \int_{r}^{\rho}\left[\frac{\operatorname{cap}_{p}\left(\Omega^{c} \cap B\left(x_{0}, t\right), B\left(x_{0}, 2 t\right)\right)}{\operatorname{cap}_{p}\left(B\left(x_{0}, t\right), B\left(x_{0}, 2 t\right)\right)}\right]^{1 /(p-1)} \frac{d t}{t}\right) .
\end{aligned}
$$

We explicitly remark that existence and uniqueness of the solution to (3.1) can be proved using the theory of monotone operators, analogously to the euclidean case (see [HKM, Appendix I]).

The proof of Theorem 3.1 will be achieved in several steps. We begin with a definition.

Definition 3.1. Let $K \subset \Omega$ be a compact set and let $f \in C_{0}^{\infty}(\Omega)$ be such that $f \equiv 1$ in $K$. The solution to the Dirichlet problem (3.1) in $\Omega \backslash K$ with boundary values $f$ is called the $(A, X, p)$-potential of $K$ in $\Omega$ and is denoted by $R(K, \Omega)$.

It is easy to check that the definition is well posed, i.e., it does not depend on the choice of the function $f$.

The following capacitary estimate of level sets of a $(A, X, p)$-potential is the first basic step in the proof of Theorem 3.1.

Lemma 3.2. Let $K \subset \Omega$ be a compact set and let $u=R(K, \Omega)$. Define, for $0<\gamma<1, K_{\gamma}=\{x \in \Omega \mid u(x) \geq \gamma\}$. Then,

$$
\frac{1}{C_{0}^{p+1}} \gamma^{p-1} \operatorname{cap}_{p}\left(K_{\gamma}, \Omega\right) \leq \operatorname{cap}_{p}(K, \Omega) \leq C_{0}^{p+1} \gamma^{p-1} \operatorname{cap}_{p}\left(K_{\gamma}, \Omega\right),
$$

where $C_{0}$ is the same constant as in (2.2).

Proof. It is completely similar to that of an analogous statement in [HKM, pg. 116] for the Euclidean case. For the sake of brevity, we confine ourselves to give an outline and refer the reader to that source for further details.

We claim that the following estimate holds:

$$
C_{0}^{-p} \int_{\{u<\gamma\}}|X u|^{p} d x \leq \gamma^{p} \operatorname{cap}_{p}\left(K_{\gamma}, \Omega\right) \leq \int_{\{u<\gamma\}}|X u|^{p} d x .
$$

In order to prove the second inequality in (3.2), fix $\varepsilon>0$ and let $K \subseteq C_{1} \subseteq$ $C_{2} \subseteq \ldots \subseteq K_{\gamma}$, where the $C_{j}$ 's are compact sets. Pick $u_{j} \in C_{0}^{\infty}(\Omega)$ such that $u_{j} \equiv 1$ in $C_{j}, 0 \leq u_{j} \leq 1$ and

$$
\int_{\Omega}\left|X u_{j}\right|^{p} d x \leq \operatorname{cap}_{p}\left(C_{j}, \Omega\right)+\varepsilon .
$$


Theorem 2.10 allows to show that

$$
\operatorname{cap}_{p}\left(C_{j}, \Omega\right) \leq \int_{\Omega \backslash C_{j}} \gamma^{-p}|X u|^{p} d x .
$$

A limiting argument yields the second part of the claim. In order to establish the first inequality in (3.2) we observe that, by a standard functional analysis argument, there exist a subsequence $\left\{v_{k}\right\}$ of $\left\{u_{j}\right\}$ and a function $v \in \stackrel{\circ}{S}^{1, p}(\Omega)$ such that $v_{k}$ converges to $v$ in $\stackrel{\circ}{S}^{1, p}(\Omega)$ and

$$
\int_{\Omega}\left|X v_{k}\right|^{p} d x \leq \operatorname{cap}_{p}\left(C_{k}, \Omega\right)+\varepsilon .
$$

This implies

$$
\int_{\Omega}|X v|^{p} d x \leq \operatorname{cap}_{p}\left(K_{\gamma}, \Omega\right)+\varepsilon .
$$

By Theorem 2.8 we may assume that $v$ is $(X, p)$-quasicontinuous and $v=1$ $(X, p)$-quasieverywhere in $K_{\gamma}$. Therefore, Theorem 2.10 guarantees that $u-\gamma v \in$ $\stackrel{\circ}{S}^{1, p}\left(\Omega \backslash K_{\gamma}\right)$. An application of Lemma 2.7 concludes the proof of the claim.

Now, observe that $\min (u / \gamma, 1)-u \in \stackrel{\circ}{S}^{1, p}(\Omega \backslash K)$ by Theorem 2.10. Keeping this in mind, together with (3.2) and the structural assumptions (2.2), we obtain

$$
\begin{aligned}
\operatorname{cap}_{p}\left(K_{\gamma}, \Omega\right) & \leq \gamma^{-p} \int_{\{u<\gamma\}}|X u|^{p} d x \leq \gamma^{-p} \sum_{j=1}^{m} \int_{\{u<\gamma\}} A_{j}(x, X u) X_{j} u d x \\
& \leq \gamma^{1-p} \sum_{j=1}^{m} \int_{\Omega} A_{j}(x, X u) X_{j} \min \left(\frac{u}{\gamma}, 1\right) d x \\
& \leq C_{0} \gamma^{1-p} \int_{\Omega}|X u|^{p} d x \leq C_{0}^{p+1} \gamma^{1-p} \operatorname{cap}_{p}(K, \Omega) .
\end{aligned}
$$

The first inequality in the lemma can be proved analogously.

Another crucial ingredient in the proof of Theorem 3.1 is an estimate, again in $(X, p)$-capacitary terms, of an "annular" condenser. As it is well known, such estimates play an important role in partial differential equations as well as in the study of quasiregular mappings.

Lemma 3.3. There exist two positive constants $R_{0}$ and $C$ such that, given $B(x, t r) \Subset \Omega, r<t r \leq R_{0}$,

$$
\frac{1}{C}|B(x, r)| r^{-p} \leq \operatorname{cap}_{p}(B(x, r), B(x, t r)) \leq C|B(x, r)| r^{-p} .
$$

The constant $C$ depends only on $\Omega$, on the vector fields $X_{1}, \ldots, X_{m}$, on $t$ and $p$. 
Proof. By Lemma 2.1, there exists a function $\psi \in C_{0}^{\infty}(B(x, t r))$ such that $0 \leq \psi \leq 1, \psi \equiv 1$ in $B(x, r)$, and $|X \psi| \leq C / r(t-1)$.

We have

$$
\operatorname{cap}_{p}(B(x, r), B(x, t r)) \leq \int_{B(x, t r)}|X \psi|^{p} d x \leq \frac{C}{r^{p}(t-1)^{p}}|B(x, t r)| .
$$

Now, by (2.6),

$$
|B(x, r)|=\left|B\left(x, \frac{1}{t} t r\right)\right| \geq C t^{-Q}|B(x, t r)|
$$

and, therefore,

$$
\operatorname{cap}_{p}(B(x, r), B(x, t r)) \leq \frac{C t^{Q}}{r^{p}(t-1)^{p}}|B(x, r)| .
$$

On the other hand, let $u \in C_{0}^{\infty}(B(x, t r)), u \geq 1$ in $B(x, r)$. The following representation formula holds (see $[\mathrm{D}])$ :

$$
|u(y)| \leq C I_{1}(|X u|)(y), \quad y \in B(x, t r) .
$$

where $I_{1}$ is as in (2.7).

Invoking Theorem 2.1, we conclude

$$
\begin{aligned}
|B(x, r)| \leq \int_{B(x, t r)}|u(y)|^{p} d y & \leq C \int_{B(x, t r)}\left|I_{1}(|X u|)(y)\right|^{p} d y \\
& \leq C(t r)^{p} \int_{B(x, t r)}|X u|^{p} d y
\end{aligned}
$$

which implies

$$
\operatorname{cap}_{p}(B(x, r), B(x, t r)) \geq C t^{-p} r^{-p}|B(x, r)| .
$$

This completes the proof.

We explicitly remark that, although Lemma 3.3 is a special case of more general and delicate $(X, p)$-capacitary estimates found in [CDG3], its short direct proof involves some tools which in the subelliptic setting are rather sophisticated.

The first important consequence of Lemmas 3.2 and 3.3 is an estimate which relates the values of a $(A, X, p)$-potential to a $(X, p)$-capacitary density formula. In the course of its proof we will need the following covering lemma, see e.g. [CW, pg. 69].

Lemma 3.4. There exist two positive constants $R_{0}$ and $K$ such that for each $r \leq R_{0}$ it is possible to find a covering $\left\{B_{d}\left(x_{j}, K r\right)\right\}$ of $\Omega$ such that the balls $\left\{B_{d}\left(x_{j}, r\right)\right\}$ are pairwise disjoint. 
Remark 3.5 By means of (2.4) and (2.5), the conclusion of Lemma 3.4 continues to hold when the metric balls $B_{d}\left(x_{j}, r\right)$ are replaced by the sets $B\left(x_{j}, r\right)$ defined in (2.3). We note explicitly that there exists a number $M$, depending only on the doubling constant $C$ in (2.6), such that any $x_{0} \in \Omega$ belongs to at most $M$ balls $B\left(x_{j}, K r\right)$.

Lemma 3.6. Let $K \subset B_{r}=B\left(x_{0}, r\right) \subseteq \Omega$ be a compact set, with $2 r \leq R_{0}$, and let $u=R\left(K, B_{2 r}\right)$. Then

$$
u(x) \geq C\left(\frac{\operatorname{cap}_{p}\left(K, B_{2 r}\right)}{\operatorname{cap}_{p}\left(B_{r}, B_{2 r}\right)}\right)^{1 /(p-1)}
$$

for each $x \in B_{r}$ and for a suitable constant $C=C\left(X_{1}, \ldots, X_{m}, \Omega, C_{0}, p\right)>0$.

Proof. Let $M=\max _{\partial B_{3 / 2 r}} u, m=\min _{\partial B_{3 / 2 r}} u$. Without loss of generality, we may assume $M>0$. By virtue of the Harnack inequality (Theorem 2.3), Lemma 3.4 and Remark 3.5, we have

$$
M \leq C m \text {. }
$$

An application of Lemma 3.2 implies

$$
\begin{aligned}
\operatorname{cap}_{p}\left(K, B_{2 r}\right) & \leq C M^{p-1} \operatorname{cap}_{p}\left(\{u \geq M\}, B_{2 r}\right) \\
& \leq C m^{p-1} \operatorname{cap}_{p}\left(\{u \geq M\}, B_{2 r}\right) .
\end{aligned}
$$

Let us show that the set $\{u \geq M\}$ is contained in $\bar{B}_{3 / 2 r}$. By contradiction, let us assume that there exists $\bar{x} \in B_{2 r} \backslash \bar{B}_{3 / 2 r}$ such that $u(\bar{x}) \geq M$. Since $\min (M-u, 0) \in \stackrel{\circ}{S}^{1, p}\left(B_{2 r} \backslash \bar{B}_{3 / 2 r}\right)$ by Theorem 2.10, Lemma 2.6 guarantees that $M \geq u$ in $B_{2 r} \backslash \bar{B}_{3 / 2 r}$. In particular, $u(\bar{x})=M$. But this contradicts the maximum principle (Theorem 2.5). Hence,

$$
\operatorname{cap}_{p}\left(\{u \geq M\}, B_{2 r}\right) \leq \operatorname{cap}_{p}\left(\bar{B}_{3 / 2 r}, B_{2 r}\right) .
$$

Lemma 3.3 yields

$$
\operatorname{cap}_{p}\left(B_{r}, B_{2 r}\right) \simeq\left|B_{r}\right| r^{-p} \simeq \operatorname{cap}_{p}\left(B_{3 / 2 r}, B_{2 r}\right) .
$$

From (3.3), (3.4), and (3.5) we infer

$$
\operatorname{cap}_{p}\left(K, B_{2 r}\right) \leq C m^{p-1} \operatorname{cap}_{p}\left(B_{r}, B_{2 r}\right) .
$$

Invoking Lemma 2.6 again, we conclude

$$
u(x) \geq C\left(\frac{\operatorname{cap}_{p}\left(K, B_{2 r}\right)}{\operatorname{cap}_{p}\left(B_{r}, B_{2 r}\right)}\right)^{1 /(p-1)} .
$$

One can infer from Lemma 3.6 the following result. 
Lemma 3.7. Let $x_{0} \in \partial \Omega, 0<\rho \leq R_{0} / 2$, and let

$$
v=1-R\left(\Omega^{c} \cap B\left(x_{0}, \rho\right), B\left(x_{0}, 2 \rho\right)\right) .
$$

Then, for each $r \leq \rho$,

$$
v \leq \exp \left(-C \int_{r}^{\rho} \varphi_{p}\left(x_{0}, \Omega^{c}, t\right)^{1 /(p-1)} \frac{d t}{t}\right)
$$

in $B\left(x_{0}, r\right)$, where $C$ is a positive constant depending only on $X_{1}, \ldots, X_{m}, \Omega, p$ and $C_{0}$ and

$$
\varphi_{p}\left(x_{0}, \Omega^{c}, t\right)=\frac{\operatorname{cap}_{p}\left(\Omega^{c} \cap B\left(x_{0}, t\right), B\left(x_{0}, 2 t\right)\right)}{\operatorname{cap}_{p}\left(B\left(x_{0}, t\right), B\left(x_{0}, 2 t\right)\right.} .
$$

Proof. Since our proof is similar to the one in [HKM, p. 120], we will omit here the lengthy details and refer to that source for a complete proof. Let $B_{\rho}=B\left(x_{0}, \rho\right)$. Fix $r \leq \rho$ and let $k \in \mathbf{N}$ be such that $2^{-k} \rho<r \leq 2^{1-k} \rho$. For $i \in \mathbf{N}$, let

$$
\begin{aligned}
& v_{i}=R\left(\Omega^{c} \cap \overline{B_{2^{1-i}} \rho}, B_{2^{2-i} \rho}\right), \\
& a_{i}=\varphi_{p}\left(x_{0}, \Omega^{c}, 2^{1-i} \rho\right) .
\end{aligned}
$$

By Lemma 3.6

$$
v_{i} \geq 1-\exp \left(-C a_{i}^{1 /(p-1)}\right) \text { in } B_{2^{1-i} \rho} .
$$

Define recursively a sequence $\left\{u_{i}\right\}$ as follows:

$$
\begin{aligned}
& u_{1}=v \\
& u_{i}=\exp \left(C a_{i-1}^{1 /(p-1)}\right) u_{i-1}, \quad i=2,3, \ldots,
\end{aligned}
$$

where $C$ is the constant appearing in Lemma 3.6. One can show by induction, using formula (3.6), that the following estimate holds:

$$
u_{i} \leq \exp \left(-C a_{i}^{1 /(p-1)}\right) \text { in } B_{2^{1-i} \rho} .
$$

We then have

$$
v \leq \exp \left(-C \sum_{i=1}^{k} a_{i}^{1 /(p-1)}\right) \text { in } B_{2^{1-k} \rho} .
$$

It is easy to infer the conclusion from this estimate.

We are now ready to prove Theorem 3.1

Proof of Theorem 3.1. Suppose, without loss of generality, $f\left(x_{0}\right)=0$. Let $\rho>0$ and let $v$ be as in Lemma 3.7. Consider the function

$$
s_{1}=v \max _{\partial \Omega} f+\underset{\partial \Omega \cap \overline{\max } \overline{B\left(x_{0}, 2 \rho\right)}}{ } f .
$$


Theorem 2.8 guarantees that $v$ can be assumed $(X, p)$-quasicontinuous in $\mathbf{R}^{n}$ and $v=1$ in $B\left(x_{0}, 2 \rho\right)^{c}$. Since $\max _{\partial \Omega} f \geq 0$, an application of Theorem 2.5 gives

$$
s_{1} \geq \max _{\partial \Omega} f \geq u \text { in } \partial B\left(x_{0}, 2 \rho\right) \cap \Omega .
$$

Invoking Theorem 2.10, we recognize that $\min \left(s_{1}, u\right)-u \in \stackrel{\circ}{S^{1, p}}\left(\Omega \cap B\left(x_{0}, 2 \rho\right)\right)$. Therefore, by Lemma 2.6,

$$
s_{1} \geq u \text { in } \Omega \cap B\left(x_{0}, 2 \rho\right) .
$$

Analogously,

$$
s_{2} \leq u \text { in } \Omega \cap B\left(x_{0}, 2 \rho\right),
$$

where

$$
s_{2}=\min _{\partial \Omega \cap \overline{B\left(x_{0}, 2 \rho\right)}} f+v \min _{\partial \Omega} f .
$$

Hence,

$$
\begin{aligned}
\operatorname{osc}\left(u, \Omega \cap B\left(x_{0}, r\right)\right) & \leq \sup _{\Omega \cap B\left(x_{0}, r\right)} s_{1}-\inf _{\Omega \cap B\left(x_{0}, r\right)} s_{2} \\
& \leq \sup _{\Omega \cap B\left(x_{0}, r\right)} v \operatorname{osc}(f, \partial \Omega)+\operatorname{osc}\left(f, \partial \Omega \cap \overline{B\left(x_{0}, 2 \rho\right)}\right) .
\end{aligned}
$$

The conclusion of the theorem follows immediately from Lemma 3.7.

At this point, we may introduce the notion of regular boundary point.

Definition 3.2. A point $x_{0} \in \partial \Omega$ is said to be regular if the solution $u$ of the Dirichlet problem (3.1) takes the limit value $f\left(x_{0}\right)$ in $x_{0}$ whenever $f \in$ $S^{1, p}(\Omega) \cap C(\bar{\Omega})$.

A sufficient condition for the regularity of boundary points can be deduced from the proof of Theorem 3.1.

Theorem 3.8. Let $x_{0} \in \partial \Omega$. If

$$
\int_{0}^{1}\left[\frac{\operatorname{cap}_{p}\left(B\left(x_{0}, t\right) \cap \Omega^{c}, B\left(x_{0}, 2 t\right)\right)}{\operatorname{cap}_{p}\left(B\left(x_{0}, t\right), B\left(x_{0}, 2 t\right)\right)}\right]^{1 /(p-1)} \frac{d t}{t}=\infty,
$$

then $x_{0}$ is regular.

Proof. Under the assumption $f\left(x_{0}\right)=0,(3.7)$ and (3.8) imply that $s_{1} \leq u \leq$ $s_{2}$ in $\Omega \cap B\left(x_{0}, 2 \rho\right)$. The conclusion follows from the fact

$$
s_{1}=\max _{\partial \Omega \cap \overline{B\left(x_{0}, 2 \rho\right)}} f, \quad s_{2}=\min _{\partial \Omega \cap \overline{B\left(x_{0}, 2 \rho\right)}} f
$$


in $B\left(x_{0}, r\right)$ for every $0<r \leq \rho$.

We now turn our attention to some applications of Theorems 3.1 and 3.8. We begin with a geometrical condition which guarantees the regularity of a boundary point.

Definition 3.3. A set $E$ is said to have a corkscrew at $x_{0} \in \partial E$ if there exist two constants $c \geq 1$ and $r_{0}>0$ such that the ball $B\left(x_{0}, r\right)$ contains a ball $B(y, r / c) \subseteq E^{c}$ for any $r \in\left(0, r_{0}\right)$.

For instance, it is easy to check that nontangentially accessible domains (NTA), in the sense of Jerison and Kenig [JK], have a corkscrew at each boundary point. NTA domains in the subelliptic context are studied in [CG].

Theorem 3.8 yields the following regularity condition.

Theorem 3.9. If $\Omega$ has a corkscrew at $x_{0} \in \partial \Omega$, then $x_{0}$ is regular.

Proof. It suffices to show that if $\Omega$ has a corkscrew at $x_{0}$, then (3.9) holds. Let $0<r<r_{0}$ be fixed. Then $B\left(x_{0}, r\right)$ contains a ball $B\left(y, \frac{r}{c}\right) \subseteq \Omega^{c}$, with $c \geq 1$ independent of $r$. Using Lemma 3.3 and formula (2.6), we obtain

$$
\begin{aligned}
\operatorname{cap}_{p}\left(\Omega^{c} \cap B\left(x_{0}, r\right), B\left(x_{0}, 2 r\right)\right) & \geq \operatorname{cap}_{p}\left(B\left(y, \frac{r}{c}\right), B\left(x_{0}, 2 r\right)\right) \\
& \geq \operatorname{cap}_{p}\left(B\left(y, \frac{r}{c}\right), B(y, 3 r)\right) \\
& \geq C\left|B\left(y, \frac{r}{c}\right)\right| r^{-p} \\
& \geq C\left|B\left(x_{0}, 2 r\right)\right| r^{-p} \\
& \geq C \operatorname{cap}_{p}\left(B\left(x_{0}, r\right), B\left(x_{0}, 2 r\right)\right) .
\end{aligned}
$$

Hence,

$$
\frac{\operatorname{cap}_{p}\left(\Omega^{c} \cap B\left(x_{0}, r\right), B\left(x_{0}, 2 r\right)\right)}{\operatorname{cap}_{p}\left(B\left(x_{0}, r\right), B\left(x_{0}, 2 r\right)\right)} \geq C>0,
$$

for $0<r<r_{0}$, and, therefore, (3.9) holds.

It is important to observe that, in general, boundary points of smooth domains in $\mathbf{R}^{n}$ are not regular. Hueber [Hu] has given examples of $C^{1, \alpha}$ domains in the Heisenberg group $\mathbf{H}^{n}$, with $n \geq 2$, which are not regular. In opposition to this, $C^{1,1}$ domains are regular in every $\mathbf{H}^{n}$ (see $[\mathrm{CG}]$ ). In $[\mathrm{HaHu}]$ the authors consider an important class of model operators, namely the sublaplacians on stratified Lie groups. If $r$ denotes the number of commutators necessary to span the tangent space, then it is proved that if either the Lie algebra has three or more generators and $r \geq 3$, or it has two generators and $r \geq 5$, then there exist $C^{\infty}$ domains having at least one irregular boundary point. 
In connection with Theorem 3.9 we mention that these examples fail to have a corkscrew at the point in question.

We conclude this paper addressing the question of the boundary Hölder continuity of solutions to (2.1). We introduce the notion of uniform $(X, p)$-thickness.

Definition 3.4. A set $E$ is said to be uniformly $(X, p)$-thick if there exist two positive constants $C$ and $R_{0}$ such that

$$
\frac{\operatorname{cap}_{p}\left(E \cap B\left(x_{0}, r\right), B\left(x_{0}, 2 r\right)\right)}{\operatorname{cap}_{p}\left(B\left(x_{0}, r\right), B\left(x_{0}, 2 r\right)\right)} \geq C
$$

for any $0<r<r_{0}$ and $x_{0} \in \partial E$.

We then have the following result:

Theorem 3.10. Suppose that $\Omega^{c}$ is uniformly $(X, p)$-thick with constants $C_{1}$ and $R_{0} \leq 1$. Let $u \in C(\bar{\Omega})$ be a solution to $(2.1)$ in $\Omega$. If

$$
|u(x)-u(y)| \leq M d(x, y)^{\delta}
$$

for all $x, y \in \partial \Omega$ and for some $M \geq 0,0<\delta \leq 1$, then there exist $M_{1} \geq 0$ and $\delta_{1}=\delta_{1}\left(Q, p, C_{0}, X_{1}, \ldots, X_{m}, \delta, C_{1}\right)>0$ such that

$$
|u(x)-u(y)| \leq M_{1} d(x, y)^{\delta_{1}}
$$

for all $x, y \in \bar{\Omega}$.

We omit the proof of Theorem 3.10, since it can be deduced with obvious adaptations from that of the analogous result for the elliptic case, see [HKM, Thm. 6.44]. We just mention that it relies on the Harnack inequality and the ensuing Hölder continuity, and on Theorem 3.1.

We point out that it is implicit in the definition of a NTA domain that the complement satisfy the uniform $(X, p)$-thickness condition, see the proof of Theorem 3.9. Therefore, the next statement is an immediate consequence of Theorem 3.10.

Corollary 3.11 Assume that $\Omega$ is a NTA domain and let $u \in C(\bar{\Omega})$ be a solution to (2.1) in $\Omega$. There (3.10) implies (3.11).

\section{REFERENCES}

[B] T. Bagby, Quasitopologies and variational approximation, J. Funct. Anal., 10 (1972), 259-268.

[CDG1] L. Capogna, D. Danielli, $\mathscr{E}$ N. Garofalo, An embedding theorem and the Harnack inequality for nonlinear subelliptic equations, Comm. P.D.E., 18 (1993), 1765-1794. 
[CDG2] L. Capogna, D. Danielli, $\mathcal{E}$ N. Garofalo, The geometric Sobolev embedding for vector fields and the isoperimetric inequality, Comm. Anal. Geom., 2 (1994), 203-215.

[CDG3] L. Capogna, D. Danielli, $\mathscr{G}$ N. Garofalo, Capacitary estimates and the local behaviour of solutions of nonlinear subelliptic equations, preprint.

[CG] L. Capogna \& N. Garofalo, NTA domains for non-commuting vector fields, (Preprint).

[CGL] G. Citti, N. Garofalo $\&$ E. Lanconelli, Harnack's inequality for sum of squares of vector fields plus a potential, Amer. Jour. of Math., 115 (1993), 699-734.

[CW] R. R. Colfman \& G. Weiss, Analyse armonique non-commutative sur certain espaces homogènes. Etudes des certaines intégrals singuliéres, Lecture Notes in Math., 242 (1971), Springer-Verlag, Berlin, New York.

[D] D. DANiELli, Formules de représentation et théorèmes d'inclusion pour des opérateurs sous-elliptiques, C.R. de l'Acad. des Sc., Paris, 314, Serie I (1992), 987-990.

[F] J. Frense, Capacity methods in the theory of partial differential equations, Jber. d. Math. Verein, 84 (1982), 1-44.

[GZ] R. GARIEPy $\&$ W. P. Ziemer, A regularity condition at the boundary for solutions of quasilinear elliptic equations, Arch. Rat. Mech. Anal., 67 (1977), 25-39.

[HaHu] W. Hansen $\& \mathrm{H}$. Hueber, The Dirichlet problem for sublaplacians on nilpotent Lie groups - Criteria for regularity, Math. Ann., 276 (1987), 537-547.

[HH] J. Heinonen $\mathscr{G}$ I. Holopainen, Quasiregular maps on Carnot groups, preprint.

[HKM] J. Heinonen, T. Kilpeläinen $\&$ O. Martio, Nonlinear potential theory of degenerate elliptic equations, Oxford Mathematical Monographs, Oxford University Press, Oxford (1993).

$[\mathrm{H}] \quad$ L. Hörmander, Hypoelliptic second order differential equations, Acta Math., 119 (1967), 147-171.

[Hu] H. HuEBER, Examples of irregular domains for some hypoelliptic differential operators, Expo. Math., 4 (1986), 189-192.

[JK] D. S. Jerison \& C. Kenig, Boundary behaviour of harmonic functions, Adv. Math., 46 (1982), 80-147.

[KR1] A. KoRÁnyi E H. M. Reimann, Horizontal normal vectors and conformal capacity of spherical rings in the Heisenberg group, Boll. Sc. Math., 2 série, 111 (1987), 3-21.

[KR2] A. KoránYi $\mathscr{G}$ H. M. Reimann, Foundations for the theory of quasiconformal mappings on the Heisenberg group, Adv. in Math. 111 (1995), $1-81$.

[M] V. G. MAz'YA, On the continuity at a boundary point of solutions of quasilinear elliptic equations, Vestnik Leningrad Univ. Math., 3 (1976), 225-242; English translation of Vestnik Leningrad Univ. Mat. Mekh. Astronom., 25 (1970), 42-55.

[NSW] A. NAgel, E. M. Stein ES S. WAinger, Balls and metrics defined by vector fields I: Basic properties, Acta Math., 155 (1985), 103-147.

[P] P. Pansu, Métriques de Carnot-Carathéodory et quasiisométries des espaces symetriques de rang un, Ann. Math., 129 (1989), 1-60.

[SC] A. SAnchez CAlle, Fundamental solutions and geometry of sum of squares of vector fields, Inv. Math., 78 (1984), 143-160. 
Department of Mathematics

Purdue University

West Lafayette, Indiana 47907

E-mail: dxd@math.purdue.edu

Received: September 30th, 1994; revised: March 8th, 1995. 